\title{
Análise da influência da variação das superfícies refletoras na disponibilidade de iluminação natural no ambiente interno e na extensão de sua profundidade
}

\section{Andrea Coelho Laranja}

Doutora em Arquitetura, Professor Adjunto do Departamento de Arquitetura e Urbanismo, Universidade Federal do Espírito Santo. Vitória [ES] Brasil. <andreacoelholaranja@gmail.com>.

\section{Nathalia Simonetti Ferreira}

Bolsista de Iniciação Científica, Laboratório de Planejamento e Projetos da Universidade Federal do Espírito Santo. Vitória [ES] Brasil. <nathaliasimonetti@hotmail.com>.

\section{Cristina Engel de Alvarez}

Doutora em Arquitetura e Urbanismo, Professor Associado II do Departamento de Arquitetura e Urbanismo, coordenadora do Laboratório de Planejamento e Projetos da Universidade Federal do Espírito Santo. Vitória [ES] Brasil. <cristina.engel@ufes.br>.

\section{Resumo}

O estudo parte da hipótese de que a disponibilidade da luz natural no ambiente interno sofre influências das características das superfícies refletoras internas. 0 objetivo é investigar o comportamento da curva isolux de um ambiente interno em função da variação de suas superfícies refletoras. A análise do ambiente em estudo é realizada por meio da construção de curvas isolux em pontos específicos do ambiente, e dos percentuais de horas em função dos intervalos das UDI (Useful Daylight Illuminance), em horários e dias do ano pré-estabelecidos. Os valores utilizados para a construção da curva isolux são gerados através das simulações do software TropLux, num ambiente de geometria retangular com abertura voltada para a orientação Norte, na cidade de Vitória (latitude $20^{\circ} 19^{\prime}$ S), utilizando céus padrões da CIE (Commission Internationale L'aclairage). Os resultados mostram que para o Céu 3, a variação das características de reflexão das superfícies refletoras internas contribuem com insignificantes aumentos na média anual de Iluminância interna. Para o Céu 7, a variação das características de reflexão das superfícies refletoras da parede e piso contribuem para pequenos aumentos na média anual de Iluminância interna no ponto 2 (1,0 m da abertura). Para o Céu 12, a variação das características de reflexão das superfícies refletoras do teto, parede e piso contribuem para pequenos aumentos na média anual de Iluminância interna somente nos pontos mais próximos da abertura, onde as superfícies refletoras do piso e teto tem contribuição mais relevante até o ponto 3 (1,5m da abertura), e a parede tem contribuição relevante somente no ponto 2 (1,0 $\mathrm{m}$ da abertura).

\section{Palavras-chave}

Superfícies refletoras, curva isolux, profundidade dos ambientes, iluminação natural.

\section{Analysis of the influence of reflecting surface changes on the availability of indoor daylighting to the extent of its depth}

\begin{abstract}
The study starts with the assumption that the availability of daylighting in the indoor environment is influenced by the characteristics of the indoor reflective surfaces. The aim is to investigate the behavior of the isolux curve of an indoor environment due to the changes in its reflective surfaces. The analysis of the environment under study is done by plotting isolux curves at specific points in the environments and determining the percentage of hours according to intervals of UDI (Useful Daylight Illuminance) in pre-established hours and days of the year. The values used to plot the isolux curve is generated through TropLux software simulations, a rectangular geometry environment with an opening facing North, in Vitória (latitude $20^{\circ} 19$ 'S) using sky patterns recognized by the CIE (Commission Internationale L'aclairage). The results show that for Sky type 3, a change in reflection characteristics of the indoor reflecting surfaces contributed little increase in annual average indoor illuminance. In Sky type 7, a change in reflection characteristics of the reflective surfaces of the wall and floor contributes to small increases in average annual internal Illuminance at point 2 (a1.0-m opening). In Sky type 12, a change in the reflection characteristics of the reflective surfaces of ceiling, wall, and floor tiles contributes to small increases in the average annual internal illuminance of only the points closest to the opening where the reflective surfaces of the floor and ceiling make a more relevant contribution to point 3 (a1.5-m opening), and the wall has a significant contribution only at point 2 (a 1.0-m opening).
\end{abstract}

\section{Keywords}

Reflective surfaces, isolux curve, depth of the environments, daylighting. 


\section{Introdução}

Estudos referentes à iluminação natural no ambiente interno, como os desenvolvidos por Hopkinson et al. (1975), Nikiforiadis \& Pitts (2003) e Laranja (2010) têm constatado que diversos são os fatores que influenciam sobre sua disponibilidade no ambiente. Uma melhor distribuição da iluminância no ambiente interno oferece ao usuário maior conforto visual para a realização das atividades, além de reduzir os gastos com iluminação artificial. Deste modo, busca-se uma uniformização desta iluminação natural ao longo do ambiente, visto que em situações de iluminação unilateral, a área próxima da abertura pode gerar situações de ofuscamento, enquanto a região mais distante pode ficar prejudicada em relação à quantidade e qualidade da iluminação recebida (Rocha, 2012).

Dentre os fatores que interferem na iluminação natural no ambiente interno, destacam-se as características das superfícies refletoras, assim como a profundidade dos ambientes internos, conforme estudos desenvolvidos por O'Connor et al. (1997), Iwashita (1999), Capeluto (2003) e Reinhart (2005). Também os trabalhos de Laranja, Gazzaneo, \& Cabús (2009), Laranja, Alvarez, \& Matarangas (2013), e Laranja, Alvarez \& Campos (2014a e 2014b) apresentam contribuições relevantes à questão.

A partir do alicerce conceitual adotado, esse estudo teve como finalidade a avaliação do comportamento da iluminação natural ao longo de um determinado ambiente interno, a partir da análise das curvas isolux. Para a efetiva avaliação de comportamento das variáveis consideradas, o ambiente simulado foi inserido em uma geometria urbana com edifícios obstruidores de dimensões fixas, sendo estabelecidas variações para a refletâncias das superfícies internas, constatando assim a real interferência que esta variação ocasiona na distribuição da luz no ambiente e sua contribuição para a homogeneização da iluminação natural do ambiente interno.

\section{Método}

Para a análise da influência da variação das superfícies refletoras foram realizadas simulações computacionais através do Software Troplux 6.07, considerando a situação geográfica da cidade de Vitória, ES (latitude 20¹9' S).

Foram inseridas no programa, por meio de coordenadas, as características adotadas para o modelo em estudo (geometria urbana e o ambiente interno) e, posteriormente, foram determinados os pontos de medição no ambiente interno, a refletância das superfícies internas e externas, a orientação das aberturas, os horários e dias das simulações, conforme procedimentos posteriormente detalhados.

Considerando os céus padrões da CIE (Commission Internationale L'aclairage), foram previamente definidas as condições de Céu 3 (encoberto), Céu 7 (parcialmente nublado) e Céu 12 (claro), uma vez que estes são compreendidos como os valores mínimo, médio e máximo, respectivamente, da média anual dos valores de iluminância interna (Laranja, 2010).

A construção de curvas isolux a partir dos dados obtidos nos pontos específicos do ambiente, assim como os percentuais de iluminância obtidos nestes pontos que são comparados aos percentuais de horas em função dos intervalos das UDI (Useful Daylight Illuminance), permitem a análise da disposição da luz natural no ambiente em estudo.

\subsection{Programa de simulação TropLux}

O software TropLux baseia-se no Metodo Monte Carlo, no Método do Raio Traçado e no conceito de coeficientes de luz natural (Araújo \& Cabús, 2007). Ele possibilita a simulação das características da iluminação natural em ambiente interno, permitindo a configuração do céu do local em que se insere o ambiente por meio da proposta da CIE (Cabús, 2006).

No input do software são inseridas por meio de coordenadas as características adotadas para o modelo em estudo: geometria urbana e o ambiente interno. Também são inseridas: a cidade em que o modelo está localizado, pontos de medição no ambiente interno, refletância das superfícies internas e externas, orientação das aberturas, horários e dias das simulações. Por fim são inseridos 
os tipos de céus que serão analisados. Por fim obtêm-se os resultados da simulação nos pontos de medição que serão posteriormente analisados.

\subsection{Avaliação da disponibilidade de iluminação natural no ambiente interno}

A disponibilidade de iluminação natural no ambiente interno é caracterizada pelas intensidades de iluminância nesse ambiente, possibilitando que atividades sejam executadas dentro dos compartimentos.

Esta disponibilidade será avaliada através dos intervalos das UDI (Useful Daylight Iluminances), apresentados por Nabil \& Mardaljevic (2006), que são classificados como: insuficientes quando os valores são inferiores a 100 lx; suficientes mas com necessidade de iluminação complementar, quando os valores estão entre 100 e 500 lx; suficientes quando os valores estão entre 500 e 2000 lx; excessivos quando os valores são superiores a 2000 lx.

Para o estudo em questão foram utilizados apenas os percentuais de horas correspondentes a faixa de intervalo suficiente das UDI, que correspondem a: $100 \leq \mathrm{UDI}<500 \mathrm{~lx}$ (suficiente, mas necessita de iluminação complementar); e $500 \leq \mathrm{UDI}<2000 \mathrm{~lx}$ (suficiente).

\subsection{Dias e horários das simulações}

Foram realizadas simulações para todos os dias do ano, compreendendo 11 horas do dia, nos períodos da manhã e da tarde, das 7 h00 às $17 \mathrm{~h} 00$.

\subsection{Características do ambiente interno adotado}

0 ambiente interno proposto para o estudo foi projetado com dimensões de 2,6m $x 3,85 \mathrm{~m} \times 2,6 \mathrm{~m}$ (largura, comprimento e pé direito respectivamente), em função de ser o modelo adotado pelo Código de Obras de Vitória (Vitória, 1998). O compartimento foi simulado considerando sua localização no primeiro pavimento tipo, que equivale ao quarto pavimento do edifício de acordo com a tipologia usual na região, ou seja, com o térreo como o primeiro pavimento; a garagem no segundo pavimento; e a área de lazer o terceiro pavimento.

A abertura na fachada foi orientada para Norte (azimute $180^{\circ}$ ), voltada para a via traçada na malha urbana, sendo composta por vidro liso transparente e dimensões de 1,14 m de largura; $1,10 \mathrm{~m}$ de altura; e peitoril de $1,10 \mathrm{~m}$, totalizando $1,25 \mathrm{~m}^{2}$ de área, condizente com a proporção mínima de 1/8 da área do piso do compartimento estabelecida pelo Código de Obras (Vitória, 1998).

\subsection{Pontos de avaliação no ambiente interno}

Foram designados sete pontos de avaliação dentro do ambiente interno de acordo com a NBR 15215-4 (Brasil, 2005) que regulamenta a distância máxima entre os pontos e a altura do ponto de avaliação da iluminação natural. Desse modo, os pontos foram alocados no plano de trabalho à $75 \mathrm{~cm}$ do piso, distando $50 \mathrm{~cm}$ entre si e alinhados no meio do ambiente (Figura 1), dispostos de maneira que o ponto 1 estivesse mais próximo da abertura, ou seja: PT $1=0,5 \mathrm{~m}$; PT $2=1,0 \mathrm{~m}$; PT $3=1,5 \mathrm{~m}$; PT $4=2,0 \mathrm{~m}$; PT $5=2,5 \mathrm{~m}$; PT $6=3,0 \mathrm{~m}$; e PT $7=3,5 \mathrm{~m}$.

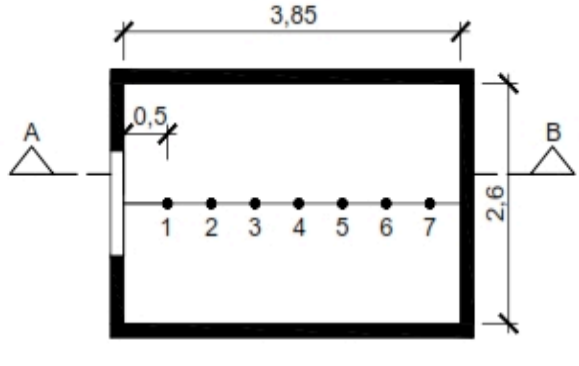

PLANTA BAIXA - SEM ESCALA

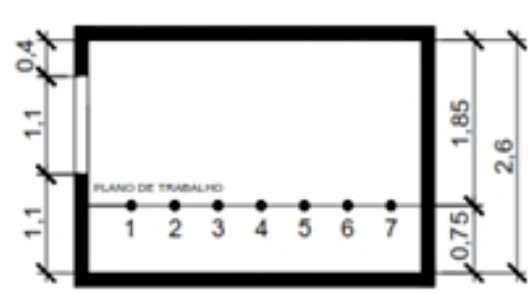

CORTE AB - SEM ESCALA

Figura 1. Planta baixa e corte $A B$ (ambos sem escala) com a demarcação dos 7 pontos analisados na simulação. 


\subsection{Características das edificações obstruidoras}

A geometria urbana foi constituída por uma via de $18 \mathrm{~m}$ de largura (rua + passeio), classificada como via "Local Principal" de acordo com o Plano Diretor Urbano de Vitória (Vitória, 2006); por três edificações obstruidoras com $45 \mathrm{~m}$ de altura (correspondente a uma edificação com aproximadamente 15 pavimentos), afastamentos laterais de $1,5 \mathrm{~m}$, e afastamento frontal e de fundos de $3 \mathrm{~m}$. A largura de via (L) e a altura da edificação obstruidora (H) compreendem uma relação de $\mathrm{H}=2,5 x \mathrm{~L}$ (Figura 2).
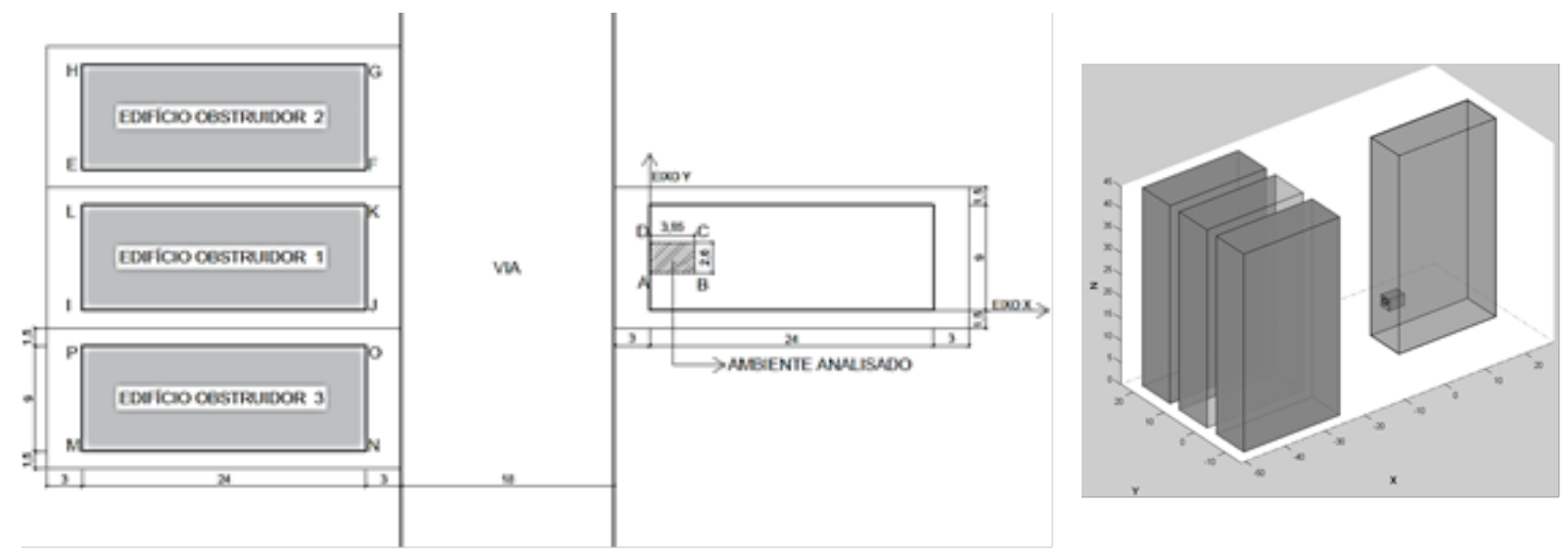

Figura 2. À esquerda, vista superior de parcela da malha urbana em que o ambiente interno em estudo foi inserido; à direita, imagem 3D gerada pelo software TropLux. Fonte: as autoras.

\subsection{Variação das superfícies refletoras do ambiente interno}

As simulações foram realizadas variando-se a refletância das superfícies internas do ambiente interno (piso, parede e teto), adotando como procedimento a fixação do valor para uma das superfícies enquanto os outros valores eram variados (Tabela 1). Esse procedimento foi repetido com todos os valores e tipos de superfícies, sendo mantida a refletância das superfícies externas das edificações obstruidoras fixas no valor de 0,4 em todas as situações.

Tabela 1. Combinações das refletâncias das superfícies internas adotadas para o ambiente analisado. No modelo 1, 2 e 3 somente o piso sofre alterações. No modelo 4, 5 e 6 somente a parede sofre alterações. No modelo 7,8 e 9 somente o teto sofre alterações.

\begin{tabular}{|c|c|c|c|c|c|c|c|c|c|}
\hline \multicolumn{10}{|c|}{ Modelos } \\
\hline $\begin{array}{c}\text { Combinações das refletâncias das superfícies internas do ambiente analisado } \\
\text { Refletância das } \\
\text { superfícies internas }\end{array}$ & 1 & 2 & 3 & 4 & 5 & 6 & 7 & 8 & 9 \\
\cline { 2 - 13 }$y$ & 0,2 & 0,4 & 0,6 & 0,2 & 0,2 & 0,2 & 0,2 & 0,2 & 0,2 \\
\hline Piso & 0,6 & 0,6 & 0,6 & 0,4 & 0,6 & 0,8 & 0,6 & 0,6 & 0,6 \\
\hline Parede & 0,8 & 0,8 & 0,8 & 0,8 & 0,8 & 0,8 & 0,4 & 0,6 & 0,8 \\
\hline
\end{tabular}

Fonte: as autoras.

\subsection{Análises de resultados}

A partir dos resultados obtidos nas simulações dos valores médios anuais de iluminância interna nos pontos localizados no plano de trabalho, curvas isolux foram criadas permitindo avaliar o comportamento da luz natural ao longo do ambiente interno considerando a variação das refletâncias das superfícies, assim como foram analisados os valores dos percentuais dentro dos intervalos das UDI estabelecidos. 
Foram formulados gráficos e tabelas a partir dos dados extraídos, considerando o Azimute $180^{\circ}$, ou seja, com a abertura orientada para Norte. As tabelas e os gráficos gerados a partir dos resultados das simulações, permitiram observar os seguintes aspectos em relação ao comportamento do ambiente: a) como ocorrem as alterações nas médias anuais de iluminância interna ao longo dos pontos distribuídos na curva isolux; b) as alterações nos percentuais de horas do dia dos intervalos das UDI ao longo dos pontos distribuídos na curva isolux; c) se é perceptível a ocorrência de um comportamento similar entre as médias de iluminância interna, bem como dos percentuais de horas do dia dos intervalos das UDI, quando comparadas as variações entre as refletâncias de piso, teto e parede.

\section{Resultados}

Através do software Troplux foi constituído o cenário para as simulações do modelo em estudo, das quais fazem parte a geometria urbana, as edificações obstruidoras, o ambiente interno, as informações sobre a cidade e os tipos de céus utilizados. A partir da adoção das características refletoras do ambiente interno, piso, teto e parede, tomando-se a orientação da abertura para Norte, foram realizadas as simulações de comportamento.

Os valores das médias anuais de iluminância interna, assim como os percentuais de horas do dia pertencentes aos intervalos das UDI caracterizados como suficientes, de cada um dos sete pontos alocados no plano de trabalho do ambiente interno, foram gerados através das simulações, cujo detalhamento segue descrito.

\subsection{Análise da iluminância interna}

De acordo com os resultados obtidos para o Céu 3 (encoberto), constatou-se que para a orientação Norte, a variação da refletância das superfícies internas do ambiente, seja no teto, parede ou piso, não ocasionam mudanças significativas nas médias anuais de iluminância interna de cada um dos pontos analisados na curva isolux, conforme verificado nos Gráficos (A, B, C) apresentados na Figura 3. Observa-se que em todos os casos, quanto mais próximo o ponto avaliado está da abertura, maiores são os valores das iluminâncias. Porém, à medida que há o afastamento desta abertura, há um perceptivel decaimento dos valores, ponto a ponto.

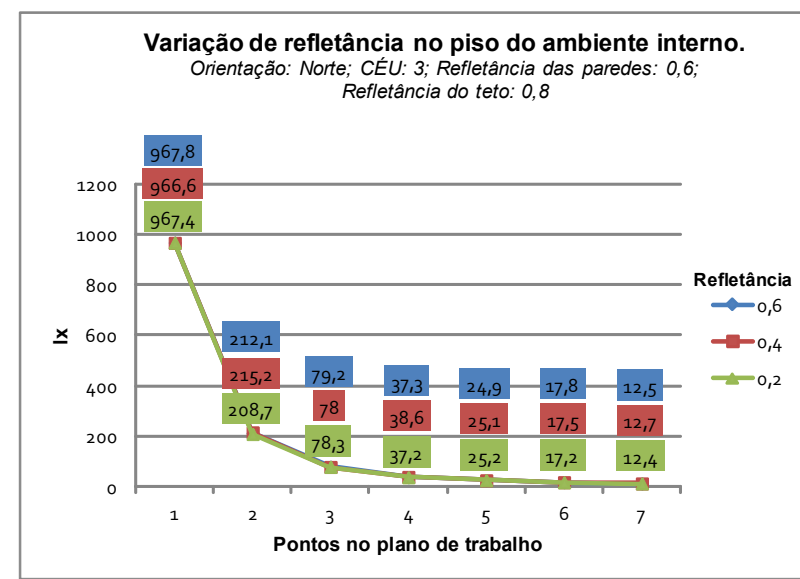

Figura 3. Variação da refletância das superfícies internas do interior do ambiente avaliado para a orientação Norte e Céu 3 (encoberto), sendo a variação : no piso (Gráfico $\mathrm{A}$, à esquerda); na parede (Gráfico $\mathrm{B}$, à direita, no alto); e no teto (Gráfico $\mathrm{C}$, à direita, em baixo), respectivamente. Fonte: as autoras.

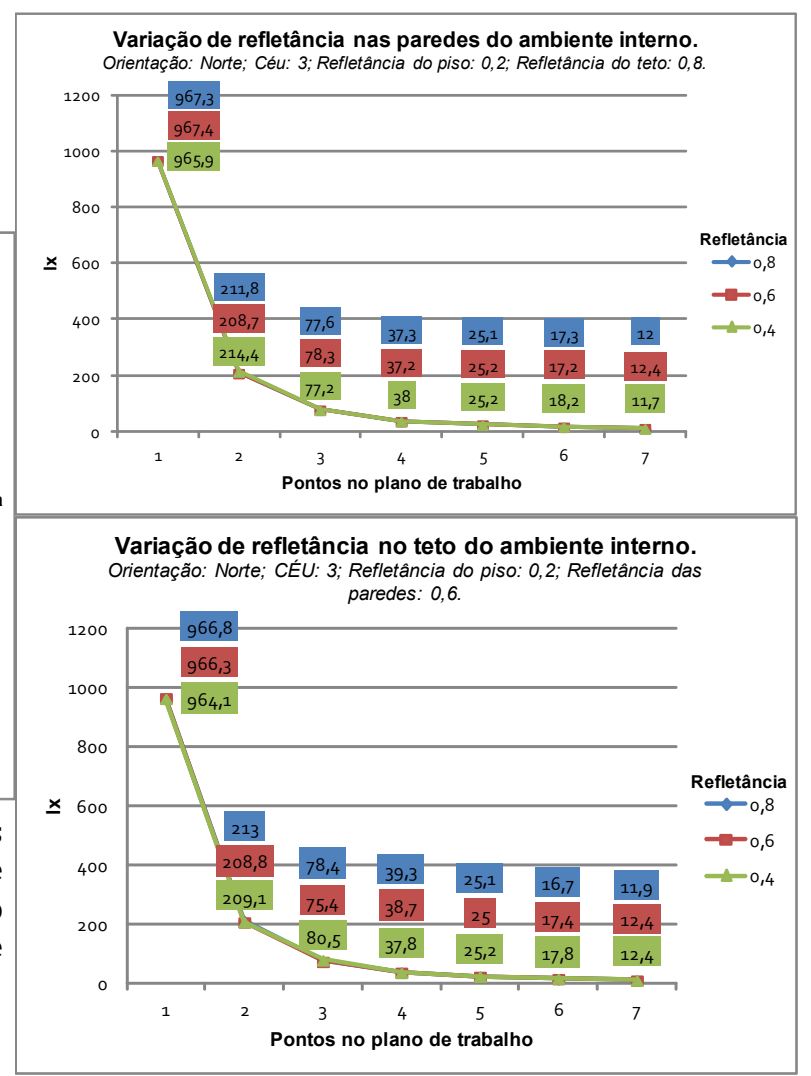


0 resultado anterior se confirma quando são observados, na Tabela 2, os percentuais de variação entre as máximas e mínimas refletâncias para piso, parede e teto. Verifica-se que, para o Céu 3, os maiores percentuais de decaimento encontram-se nos pontos mais próximos da abertura quando são variadas as refletâncias somente de piso e parede. Estes valores são, porém, ainda pequenos, com variações percentuais que não ultrapassam os $10 \%$. Desta forma conclui-se que, para o Céu 3, a variação das características de reflexão das superfícies refletoras do piso, parede e teto contribuem para aumentos insignificantes na média anual de Iluminância interna.

Tabela 2. Variações percentuais entre as máximas e mínimas refletâncias para piso, parede e teto, para o Céu 3 (encoberto) e orientação Norte.

\begin{tabular}{|c|c|c|c|c|c|c|c|}
\hline \multicolumn{7}{|c|}{ Variações percentuais entre as máximas e mínimas refletâncias para piso, teto e parede } \\
Orientação: Norte / Céu 3 \\
\hline \multirow{7}{*}{$\begin{array}{c}\text { Variação da refletância das } \\
\text { superfícies internas }\end{array}$} & 1 & 2 & 3 & 4 & 5 & 6 & 7 \\
\cline { 2 - 9 } & \multicolumn{7}{c|}{ Pontos de avaliação no ambiente } \\
\hline 0,4 e 0,2 (Piso) & $3,3 \%$ & $5,7 \%$ & $3,3 \%$ & $0,8 \%$ & $0,0 \%$ & $1,0 \%$ & $0,7 \%$ \\
\hline 0,6 e 0,4 (Parede) & $1,1 \%$ & $6,5 \%$ & $2,9 \%$ & $1,5 \%$ & $0,2 \%$ & $0,3 \%$ & $0,3 \%$ \\
\hline 0,8 e 0,6 (Teto) & $0,0 \%$ & $1,2 \%$ & $1,6 \%$ & $2,0 \%$ & $0,2 \%$ & $1,1 \%$ & $0,6 \%$ \\
\hline
\end{tabular}

Fonte: as autoras.

A Tabela 3 apresenta o percentual de variação das médias anuais de iluminância interna, ponto a ponto, quando são alteradas as refletâncias das superfícies do ambiente interno. Observam-se que as médias anuais de iluminância variam entre $27 \%$ e $78 \%$ ao longo da profundidade do ambiente para todas as variações de refletância (piso, teto e parede). 0 maior decaimento percentual ocorre até o ponto 4 (2,0 $\mathrm{m}$ da abertura). A partir deste ponto, os decaimentos percentuais apresentam-se com valores próximos entre si. Conclui-se desta forma que, a variação nas refletâncias de piso, teto e parede, ocasionam perdas na iluminação de forma similar na curva isolux.

Tabela 3. Porcentagem de variação da média anual de iluminância interna média para o Céu 3 (encoberto) em função dos pontos avaliados ao longo da profundidade do ambiente interno.

Percentual de variação dos valores das médias anuais de iluminância interna (\%) para o Céu 3 (encoberto) - ponto a ponto

\begin{tabular}{|c|c|c|c|c|c|c|c|c|}
\hline \multirow{2}{*}{ Modelos } & \multicolumn{7}{c|}{ Pontos de avaliação no ambiente } \\
\cline { 2 - 10 } & 1 & 2 & 3 & 4 & 5 & 6 & 7 \\
\hline \multirow{2}{*}{$\begin{array}{c}\text { Somente o } \\
\text { Piso sofre } \\
\text { alterações }\end{array}$} & 1 & - & $-78,4 \%$ & $-62,5 \%$ & $-52,5 \%$ & $-32,3 \%$ & $-31,7 \%$ & $-27,9 \%$ \\
\cline { 2 - 10 } & 3 & - & $-77,7 \%$ & $-63,8 \%$ & $-50,5 \%$ & $-35,0 \%$ & $-30,3 \%$ & $-27,4 \%$ \\
\hline \multirow{2}{*}{$\begin{array}{c}\text { Somente a } \\
\text { Parede } \\
\text { sofre }\end{array}$} & 4 & - & $-78,1 \%$ & $-62,7 \%$ & $-52,9 \%$ & $-33,2 \%$ & $-28,5 \%$ & $-29,8 \%$ \\
\cline { 2 - 10 } alterações & 6 & - & $-77,8 \%$ & $-64,0 \%$ & $-50,8 \%$ & $-33,7 \%$ & $-27,8 \%$ & $-35,7 \%$ \\
\hline \multirow{2}{*}{$\begin{array}{c}\text { Somente o } \\
\text { Teto sofre } \\
\text { alterações }\end{array}$} & 7 & - & $-78,1 \%$ & $-63,4 \%$ & $-51,9 \%$ & $-32,7 \%$ & $-31,1 \%$ & $-30,6 \%$ \\
\cline { 2 - 10 }$y$ & 9 & - & $-78,3 \%$ & $-61,5 \%$ & $-53,0 \%$ & $-33,3 \%$ & $-29,4 \%$ & $-30,3 \%$ \\
\hline
\end{tabular}

Fonte: as autoras. 
De acordo com os resultados obtidos, para o Céu 7 (parcialmente nublado), constatou-se que para a orientação Norte, a partir do ponto $3(1,5 \mathrm{~m})$ a variação da refletância das superfícies internas do ambiente não ocasionou mudanças significativas nas médias anuais de iluminância interna em cada um dos pontos analisados na curva isolux, conforme verificado nos Gráficos (A, B, C) apresentados na Figura 4. Observa-se que em todos os casos, quanto mais próximo o ponto avaliado está da abertura, maiores são os valores das iluminâncias. Porém, à medida que há o afastamento desta abertura, há um perceptível decaimento dos valores, ponto a ponto.
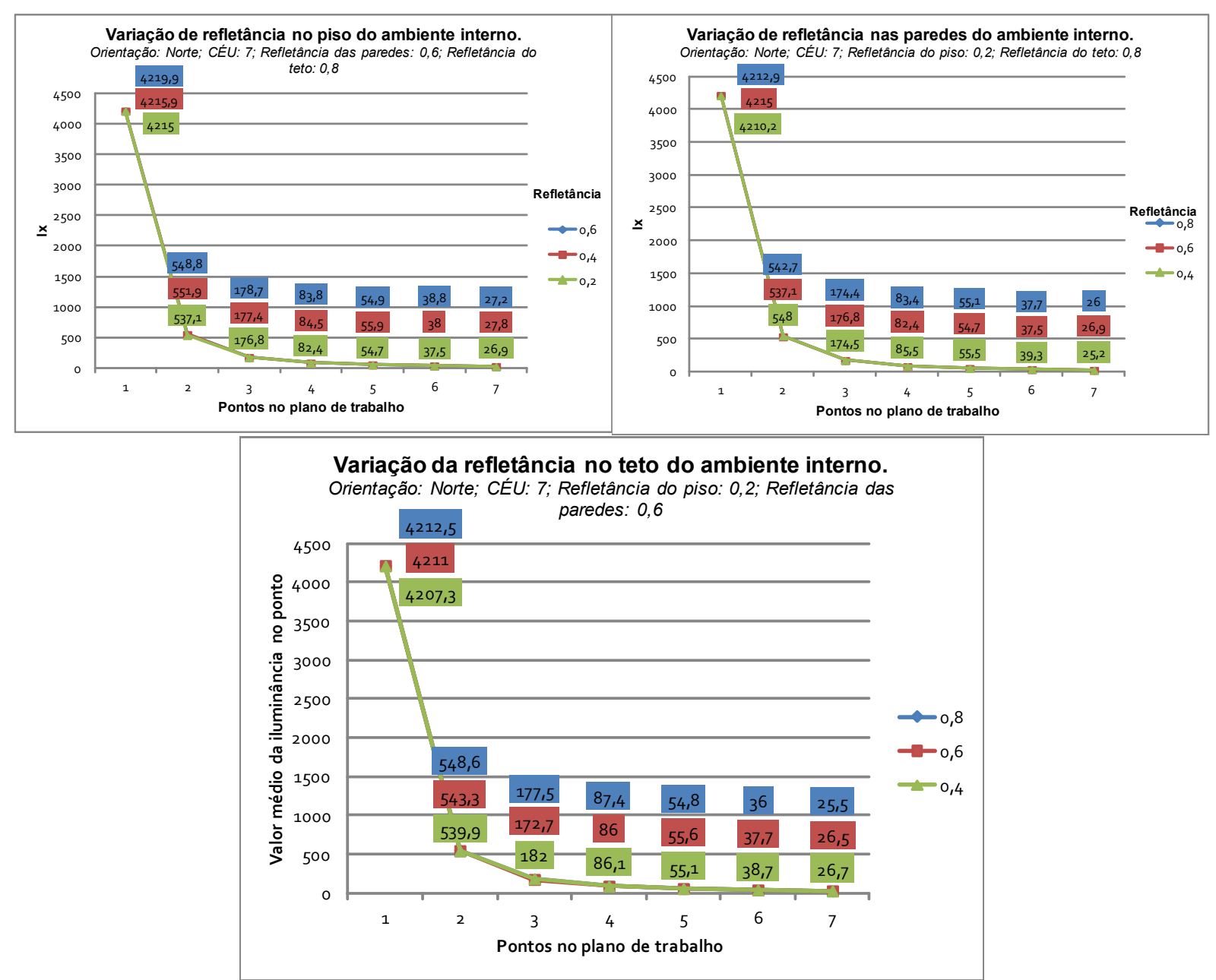

Figura 4. Variação da refletância das superfícies internas do interior do ambiente para a orientação Norte e Céu 7 (parcialmente nublado), sendo a variação : no piso (Gráfico A, à esquerda, no alto); na parede (Gráfico B, à direita, no alto); e no teto (Gráfico C, no centro, embaixo), respectivamente.

Fonte: as autoras.

O resultado anterior se confirma quando são observados, na Tabela 4, os percentuais de variação entre as máximas e mínimas refletâncias para piso, parede e teto. Verifica-se que os maiores percentuais de decaimento encontram-se nos pontos mais próximos da abertura. Estes valores são, porém, ainda pequenos, com variações percentuais que não ultrapassam os $10 \%$, exceto pelo percentual de $10,9 \%$ e $14,8 \%$ encontrado no ponto 2 , quando variam-se as refletâncias do piso e parede. Desta forma concluise que, para o Céu 7, a variação das características de reflexão das superfícies refletoras da parede e piso contribuem para pequenos aumentos na média anual de Iluminância interna, onde somente no ponto 2 (1,0 m da abertura) a contribuição da superfície do piso e parede é mais eficaz.

A Tabela 5 apresenta o percentual de variação das médias anuais de iluminância interna ponto a ponto, quando são alteradas as refletâncias das superfícies internas. Observam-se que as médias anuais de iluminância variam entre $27 \%$ e $87 \%$ ao longo da profundidade do ambiente para todas as refletâncias. 0 maior decaimento de percentual ocorre até o ponto 4 (2,0 $\mathrm{m}$ da abertura) e, a partir deste ponto, os decaimentos percentuais ocorrem, mas de maneira mais amena. Conclui-se desta forma, que a variação nas refletâncias de piso, teto e parede ocasionam perdas na iluminação de forma similar na curva isolux. 
Tabela 4. Variações percentuais entre as máximas e mínimas refletâncias para piso, parede e teto, para o Céu 7 (parcialmente nublado) e orientação Norte.

\begin{tabular}{|c|c|c|c|c|c|c|c|}
\hline \multicolumn{8}{|c|}{$\begin{array}{c}\text { Variações percentuais entre as máximas e mínimas refletâncias para piso, teto e parede } \\
\text { Orientação: Norte / Céu } 7\end{array}$} \\
\hline \multirow{2}{*}{$\begin{array}{l}\text { Variação da refletância das } \\
\text { superfícies internas }\end{array}$} & \multicolumn{7}{|c|}{ Pontos de avaliação no ambiente } \\
\hline & 1 & 2 & 3 & 4 & 5 & 6 & 7 \\
\hline 0,4 e 0,2 (Piso) & $7,7 \%$ & $10,9 \%$ & $7,5 \%$ & $3,7 \%$ & $0,8 \%$ & $1,8 \%$ & $1,7 \%$ \\
\hline 0,6 e 0,4 (Parede) & $4,9 \%$ & $14,8 \%$ & $4,7 \%$ & $1,5 \%$ & $1,2 \%$ & $0,5 \%$ & $1,3 \%$ \\
\hline 0,8 e 0,6 (Teto) & $7,4 \%$ & $6,1 \%$ & $4,3 \%$ & $4,0 \%$ & $0,3 \%$ & $1,3 \%$ & $1,7 \%$ \\
\hline
\end{tabular}

Fonte: as autoras.

Tabela 5. Porcentagem de variação da média anual de iluminância interna média para o Céu 7 (parcialmente nublado) em função dos pontos avaliados ao longo da profundidade do ambiente interno.

\begin{tabular}{|c|c|c|c|c|c|c|c|c|}
\hline \multicolumn{9}{|c|}{$\begin{array}{l}\text { Percentual de variação dos valores das médias anuais de iluminância interna (\%) de para o } \\
\text { Céu } 7 \text { (parcialmente nublado) - ponto a ponto }\end{array}$} \\
\hline \multirow{2}{*}{\multicolumn{2}{|c|}{ Modelos }} & \multicolumn{7}{|c|}{ Pontos de avaliação no ambiente } \\
\hline & & 1 & 2 & 3 & 4 & 5 & 6 & 7 \\
\hline \multirow{3}{*}{$\begin{array}{l}\text { Somente o } \\
\text { Piso sofre } \\
\text { alterações }\end{array}$} & 1 & - & $-87,3 \%$ & $-67,1 \%$ & $-53,4 \%$ & $-33,6 \%$ & $-31,4 \%$ & $-28,3 \%$ \\
\hline & 2 & - & $-86,9 \%$ & $-67,9 \%$ & $-52,4 \%$ & $-33,8 \%$ & $-32,0 \%$ & $-26,8 \%$ \\
\hline & 3 & - & $-87,0 \%$ & $-67,4 \%$ & $-53,1 \%$ & $-34,5 \%$ & $-29,3 \%$ & $-29,9 \%$ \\
\hline \multirow{3}{*}{$\begin{array}{c}\text { Somente a } \\
\text { Parede } \\
\text { sofre } \\
\text { alterações }\end{array}$} & 4 & - & $-87,0 \%$ & $-68,2 \%$ & $-51,0 \%$ & $-35,1 \%$ & $-29,2 \%$ & $-35,9 \%$ \\
\hline & 5 & - & $-87,3 \%$ & $-67,1 \%$ & $-53,4 \%$ & $-33,6 \%$ & $-31,4 \%$ & $-28,3 \%$ \\
\hline & 6 & - & $-87,1 \%$ & $-67,9 \%$ & $-52,2 \%$ & $-33,9 \%$ & $-31,6 \%$ & $-31,0 \%$ \\
\hline \multirow{3}{*}{$\begin{array}{l}\text { Somente o } \\
\text { Teto sofre } \\
\text { alterações }\end{array}$} & 7 & - & $-87,2 \%$ & $-66,3 \%$ & $-52,7 \%$ & $-36,0 \%$ & $-29,8 \%$ & $-31,0 \%$ \\
\hline & 8 & - & $-87,1 \%$ & $-68,2 \%$ & $-50,2 \%$ & $-35,3 \%$ & $-32,2 \%$ & $-29,7 \%$ \\
\hline & 9 & - & $-87,0 \%$ & $-67,6 \%$ & $-50,8 \%$ & $-37,3 \%$ & $-34,3 \%$ & $-29,2 \%$ \\
\hline
\end{tabular}

Fonte: as autoras.

De acordo com os resultados obtidos para o Céu 12 (claro), constata-se com os Gráficos (A, B, C) apresentados na Figura 5, que, para a orientação Norte, a partir do ponto $4(2,0 \mathrm{~m})$ a variação da refletância das superfícies internas do ambiente (piso, teto ou parede) não ocasionam mudanças significativas nas médias anuais de iluminância interna em cada um dos pontos analisados na curva isolux. Observa-se que em todos os casos, quanto mais próximo o ponto avaliado está da abertura, maiores são os valores das iluminâncias. Porém, à medida que há o afastamento desta abertura, há um perceptível decaimento dos valores, ponto a ponto.

0 resultado anterior se confirma quando são observados, na Tabela 6, os percentuais de variação das refletâncias para piso, teto e parede. Desta forma conclui-se que, para o Céu 12, a variação das características de reflexão das superfícies refletoras do teto, parede e piso contribuem para pequenos aumentos na média anual de Iluminância interna somente nos pontos mais próximos da abertura. Variando-se a característica de reflexão do teto sua contribuição é relevante até o ponto 3 (1,5 m da abertura), variando-se a característica de reflexão do piso sua contribuição é relevante até até o ponto 4 (2,0 m da abertura), variando-se a característica de reflexão da parede sua contribuição é relevante no ponto 2 (1,0 $\mathrm{m}$ da abertura). A medida que os pontos se afastam da abertura as variações na média anual de iluminância interna torna-se insignificantes. 

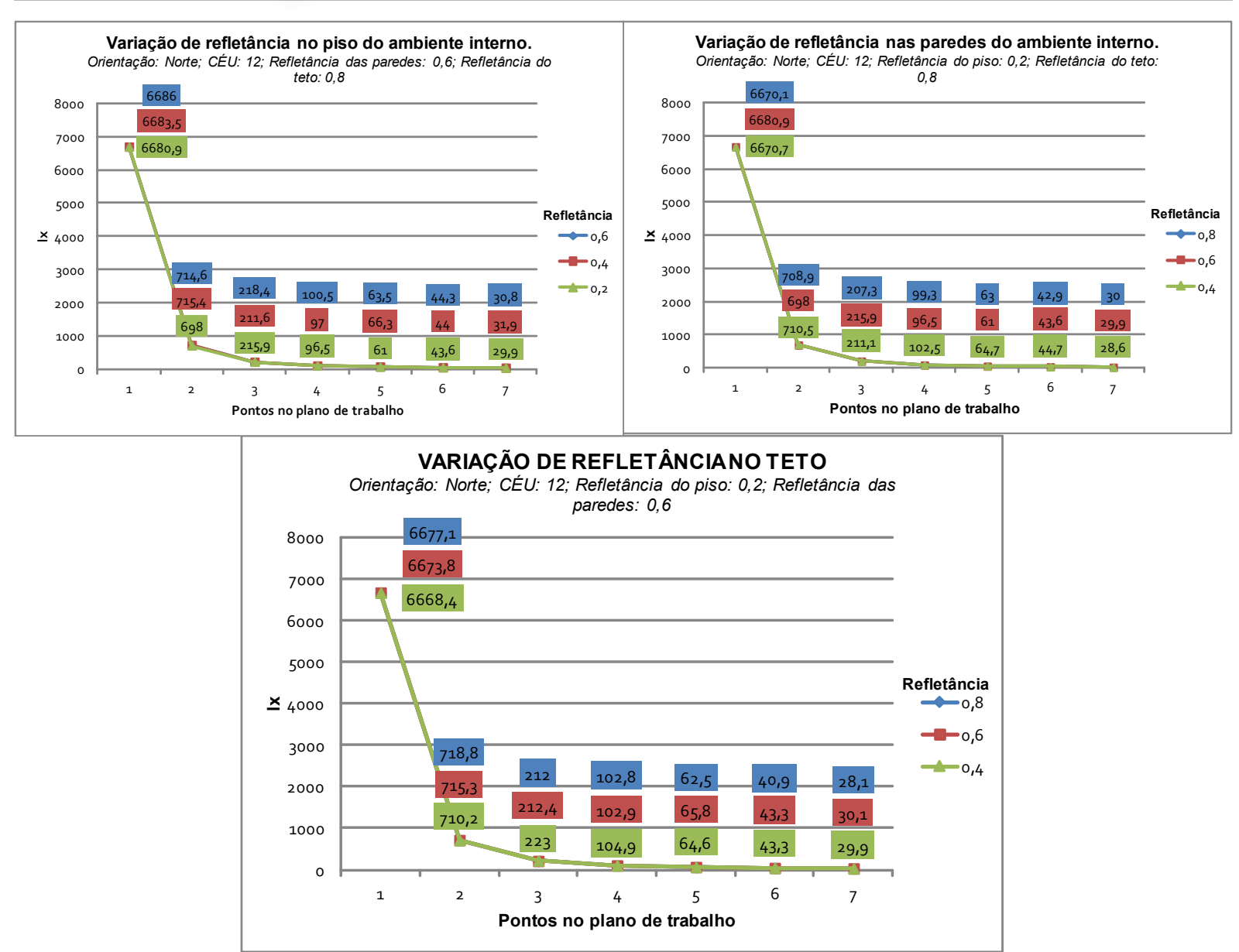

Figura 5. Variação da refletância das superfícies internas do interior do ambiente para a orientação Norte e Céu 12 (claro), sendo a variação: no piso (Gráfico A, à esquerda, no alto); na parede (Gráfico B, à direita, no alto); e no teto (Gráfico C, no centro, embaixo), respectivamente.

Fonte: as autoras.

Tabela 6. Variações percentuais entre as máximas e mínimas refletâncias para piso, parede e teto, para o Céu 12 (claro) e orientação Norte.

\begin{tabular}{|c|c|c|c|c|c|c|}
\hline \multicolumn{7}{|c|}{ Variações percentuais entre as máximas e mínimas refletâncias para piso, teto e parede } \\
Orientação: Norte / Céu 12
\end{tabular}

Fonte: as autoras.

A Tabela 7 apresenta o percentual de variação das médias anuais de iluminância interna, ponto a ponto, quando são alteradas as refletâncias das superfícies internas. 0 maior decaimento percentual ocorre até o ponto 4 (2,0 $\mathrm{m}$ da abertura), quando então os decaimentos percentuais apresentam-se com valores próximos entre si. Conclui-se desta forma que, a variação nas refletâncias de piso, teto e parede, ocasionam perdas na iluminação de forma similar na curva isolux. 
Tabela 7. Porcentagem de variação da média anual de iluminância interna média para o Céu 7 (parcialmente nublado) em função dos pontos avaliados ao longo da profundidade do ambiente interno.

\begin{tabular}{|c|c|c|c|c|c|c|c|c|}
\hline \multicolumn{9}{|c|}{$\begin{array}{l}\text { Percentual de variação dos valores das médias anuais de iluminância interna (\%) de para } 0 \\
\text { Céu } 12 \text { (claro) - ponto a ponto }\end{array}$} \\
\hline \multirow{2}{*}{\multicolumn{2}{|c|}{ Modelos }} & \multicolumn{7}{|c|}{ Pontos de avaliação no ambiente } \\
\hline & & 1 & 2 & 3 & 4 & 5 & 6 & 7 \\
\hline \multirow{3}{*}{$\begin{array}{l}\text { Somente o } \\
\text { Piso sofre } \\
\text { alterações }\end{array}$} & 1 & - & $-89,6 \%$ & $-69,1 \%$ & $-55,3 \%$ & $-36,8 \%$ & $-28,5 \%$ & $-31,4 \%$ \\
\hline & 2 & - & $-89,3 \%$ & $-70,4 \%$ & $-54,2 \%$ & $-31,6 \%$ & $-33,6 \%$ & $-27,5 \%$ \\
\hline & 3 & - & $-89,3 \%$ & $-69,4 \%$ & $-54,0 \%$ & $-36,8 \%$ & $-30,2 \%$ & $-30,5 \%$ \\
\hline \multirow{3}{*}{$\begin{array}{l}\text { Somente a } \\
\text { Parede } \\
\text { sofre } \\
\text { alterações }\end{array}$} & 4 & - & $-89,3 \%$ & $-70,3 \%$ & $-51,4 \%$ & $-36,9 \%$ & $-30,9 \%$ & $-36,0 \%$ \\
\hline & 5 & - & $-89,6 \%$ & $-69,1 \%$ & $-55,3 \%$ & $-36,8 \%$ & $-28,5 \%$ & $-31,4 \%$ \\
\hline & 6 & - & $-89,4 \%$ & $-70,8 \%$ & $-52,1 \%$ & $-36,6 \%$ & $-31,9 \%$ & $-30,1 \%$ \\
\hline \multirow{3}{*}{$\begin{array}{l}\text { Somente o } \\
\text { Teto sofre } \\
\text { alterações }\end{array}$} & 7 & - & $-89,3 \%$ & $-68,6 \%$ & $-53,0 \%$ & $-38,4 \%$ & $-33,0 \%$ & $-30,9 \%$ \\
\hline & 8 & - & $-89,3 \%$ & $-70,3 \%$ & $-51,6 \%$ & $-36,1 \%$ & $-34,2 \%$ & $-30,5 \%$ \\
\hline & 9 & - & $-89,2 \%$ & $-70,5 \%$ & $-51,5 \%$ & $-39,2 \%$ & $-34,6 \%$ & $-31,3 \%$ \\
\hline
\end{tabular}

Fonte: as autoras.

\subsection{Análise dos percentuais de horas do dia enquadrados nos intervalos das UDI}

A Tabela 8 mostra que para o Céu 3 (encoberto), somente até o ponto 3 (1,5 $\mathrm{m}$ da abertura) há percentuais de horas no intervalo $100 \leq \mathrm{UDI}<500$ lx. Nota-se também um comportamento similar quando se alteram as superfícies refletoras do teto, parede e piso. Nos modelos propostos, os menores percentuais ocorrem no ponto 1 (cerca de 15\% das horas diárias apresentam valores suficientes mas com necessidade de iluminação complementar), enquanto os maiores percentuais ocorrem no ponto 2 (em torno de $89 \%$ ).

Tabela 8. Porcentagem diária que se insere no intervalo de UDI (Useful Daylight Illuminances) entre 100 a 500 lx para o Céu 3 (encoberto) em função da refletância das superfícies do ambiente interno.

\begin{tabular}{|c|c|c|c|c|c|c|c|c|}
\hline \multicolumn{9}{|c|}{ Percentual de horas diárias no intervalo entre $100 \leq \mathrm{UDI}<500$ lx para o Céu 3 (encoberto) } \\
\hline \multirow{2}{*}{\multicolumn{2}{|c|}{ Modelos }} & \multicolumn{7}{|c|}{ Pontos de avaliação no ambiente } \\
\hline & & 1 & 2 & 3 & 4 & 5 & 6 & 7 \\
\hline \multirow{3}{*}{ Piso } & 1 & $15,3 \%$ & $88,6 \%$ & $29,2 \%$ & $0 \%$ & $0 \%$ & $0 \%$ & $0 \%$ \\
\hline & 2 & $15,3 \%$ & $89,0 \%$ & $29,0 \%$ & $0 \%$ & $0 \%$ & $0 \%$ & $0 \%$ \\
\hline & 3 & $15,2 \%$ & $88,1 \%$ & $30,0 \%$ & $0 \%$ & $0 \%$ & $0 \%$ & $0 \%$ \\
\hline \multirow{3}{*}{ Parede } & 4 & $15,3 \%$ & $89,0 \%$ & $28,1 \%$ & $0 \%$ & $0 \%$ & $0 \%$ & $0 \%$ \\
\hline & 5 & $15,3 \%$ & $88,6 \%$ & $29,2 \%$ & $0 \%$ & $0 \%$ & $0 \%$ & $0 \%$ \\
\hline & 6 & $15,3 \%$ & $88,8 \%$ & $28,6 \%$ & $0 \%$ & $0 \%$ & $0 \%$ & $0 \%$ \\
\hline \multirow{3}{*}{ Teto } & 7 & $15,4 \%$ & $88,6 \%$ & $31,1 \%$ & $0 \%$ & $0 \%$ & $0 \%$ & $0 \%$ \\
\hline & 8 & $15,3 \%$ & $88,6 \%$ & $26,5 \%$ & $0 \%$ & $0 \%$ & $0 \%$ & $0 \%$ \\
\hline & 9 & $15,3 \%$ & $88,9 \%$ & $29,3 \%$ & $0 \%$ & $0 \%$ & $0 \%$ & $0 \%$ \\
\hline
\end{tabular}

Fonte: as autoras. 
É possível constatar na Tabela 9, que para todos os modelos de refletância testados para o Céu 3 (encoberto), só existem percentuais de horas no intervalo das UDI entre 500 lx e 2000 lx, no ponto 1 (0,5 $\mathrm{m}$ da abertura), mantendo um comportamento similar quando se alteram as superfícies refletoras do teto, parede e piso. Ou seja, a variação das refletâncias internas não ocasiona mudanças significativas neste intervalo de valores das UDI.

Tabela 9. Porcentagem diária em que ocorre o intervalo de UDI (Useful Daylight Illuminances) entre 500 lx a 2000 lx para o Céu 3 (encoberto) em função da refletância das superfícies do ambiente interno.

\begin{tabular}{|c|c|c|c|c|c|c|c|}
\hline \multirow{2}{*}{ Percentual de horas diárias no intervalo entre 500 $\mathbf{U}$ UDI<2000 lx para o Céu 3 (encoberto) } \\
\hline \multirow{2}{*}{ Modelos } & 1 & 2 & 3 & 4 & 5 & 6 & 7 \\
\cline { 2 - 8 } & $84,7 \%$ & $0 \%$ & $0 \%$ & $0 \%$ & $0 \%$ & $0 \%$ & $0 \%$ \\
\hline $\begin{array}{l}\text { Todos os } \\
\text { modelos }\end{array}$ & $8 \%$ Pontos de avaliação no ambiente \\
\hline
\end{tabular}

Fonte: as autoras.

A Tabela 10 mostra que para o Céu 7 (parcialmente nublado), somente até o ponto 5 (2,5 $\mathrm{m}$ da abertura) verificam-se percentuais de horas inseridas no intervalo $100 \leq \mathrm{UDI}<500 \mathrm{~lx}$. Nota-se também um comportamento similar quando se alteram as superfícies refletoras do teto, parede e piso. Em todos os modelos propostos os valores percentuais mais elevados encontram-se no ponto 3 (1,5 $\mathrm{m}$ da abertura), e reduzem a medida que os pontos se distanciam da abertura. Isto ocorre porque o ponto $1(0,5 \mathrm{~m}$ da abertura) e ponto $2(1,0 \mathrm{~m}$ da abertura) possuem a complementação dos percentuais das UDI no intervalo $500 \leq \mathrm{UDI}<2000 \mathrm{~lx}$.

Tabela 10. Porcentagem diária em que ocorre o intervalo de UDI (Useful Daylight Illuminances) entre 100 lx a 500 lx para o Céu 7 (parcialmente nublado) em função da refletância das superfícies do ambiente interno.

\begin{tabular}{|c|c|c|c|c|c|c|c|c|}
\hline \multicolumn{9}{|c|}{ Percentual de horas diárias no intervalo entre $100 \leq \mathrm{UDI}<500$ lx para o Céu 7 (parcialmente nublado } \\
\hline \multirow{2}{*}{\multicolumn{2}{|c|}{ Modelos }} & \multicolumn{7}{|c|}{ Pontos de avaliação no ambiente } \\
\hline & & 1 & 2 & 3 & 4 & 5 & 6 & 7 \\
\hline \multirow{3}{*}{$\begin{array}{l}\text { Somente o } \\
\text { Piso sofre } \\
\text { alterações }\end{array}$} & 1 & $6,2 \%$ & $41,5 \%$ & $82,3 \%$ & $33,7 \%$ & $1,6 \%$ & $0 \%$ & $0 \%$ \\
\hline & 2 & $6,2 \%$ & $40,2 \%$ & $81,5 \%$ & $35,6 \%$ & $3,1 \%$ & $0 \%$ & $0 \%$ \\
\hline & 3 & $6,1 \%$ & $41,8 \%$ & $82,8 \%$ & $33,7 \%$ & $1,6 \%$ & $0 \%$ & $0 \%$ \\
\hline \multirow{3}{*}{$\begin{array}{l}\text { Somente a } \\
\text { Parede } \\
\text { sofre } \\
\text { alterações }\end{array}$} & 4 & $6,2 \%$ & $40,6 \%$ & $82,4 \%$ & $38,6 \%$ & $2,8 \%$ & $0 \%$ & $0 \%$ \\
\hline & 5 & $6,2 \%$ & $41,5 \%$ & $82,3 \%$ & $33,7 \%$ & $1,6 \%$ & $0 \%$ & $0 \%$ \\
\hline & 6 & $6,2 \%$ & $44,6 \%$ & $81,8 \%$ & $34,2 \%$ & $3,0 \%$ & $0 \%$ & $0 \%$ \\
\hline \multirow{3}{*}{$\begin{array}{l}\text { Somente o } \\
\text { Teto sofre } \\
\text { alterações }\end{array}$} & 7 & $6,2 \%$ & $40,6 \%$ & $83,8 \%$ & $34,3 \%$ & $2,6 \%$ & $0 \%$ & $0 \%$ \\
\hline & 8 & $6,2 \%$ & $41,1 \%$ & $81,5 \%$ & $37,2 \%$ & $4,0 \%$ & $0 \%$ & $0 \%$ \\
\hline & 9 & $6,2 \%$ & $40,3 \%$ & $82,4 \%$ & $37,2 \%$ & $1,6 \%$ & $0 \%$ & $0 \%$ \\
\hline
\end{tabular}

Fonte: as autoras.

É possível constatar, de acordo com a Tabela 11, que para todos os modelos de refletância testados para o Céu 7 (parcialmente nublado), só existem percentuais de horas inseridos no intervalo das UDI entre $500 \mathrm{~lx}$ e $2000 \mathrm{~lx}$ até o ponto 2 (1,0 $\mathrm{m}$ da abertura), mantendo um comportamento similar quando se alteram as superfícies refletoras do teto, parede e piso. $\mathrm{Ou}$ seja, a variação das refletâncias internas não ocasiona mudanças significativas neste intervalo de valores das UDI. 
Tabela 11. Porcentagem diária em que ocorre o intervalo de UDI (Useful Daylight Illuminances) entre 500 lx a 2000 lx para o Céu 7 (parcialmente nublado) em função da refletância das superfícies do ambiente interno.

\begin{tabular}{|c|c|c|c|c|c|c|c|c|}
\hline \multicolumn{9}{|c|}{ Percentual de horas diárias no intervalo entre $500 \leq \mathrm{UDI}<2000$ lx para o Céu 7 (parcialmente nublado } \\
\hline \multirow{2}{*}{\multicolumn{2}{|c|}{ Modelos }} & \multicolumn{7}{|c|}{ Pontos de avaliação no ambiente } \\
\hline & & 1 & 2 & 3 & 4 & 5 & 6 & 7 \\
\hline \multirow{3}{*}{$\begin{array}{l}\text { Somente o } \\
\text { Piso sofre } \\
\text { alterações }\end{array}$} & 1 & $34,1 \%$ & $53,6 \%$ & $0 \%$ & $0 \%$ & $0 \%$ & $0 \%$ & $0 \%$ \\
\hline & 2 & $34,1 \%$ & $55,1 \%$ & $0 \%$ & $0 \%$ & $0 \%$ & $0 \%$ & $0 \%$ \\
\hline & 3 & $34,1 \%$ & $53,2 \%$ & $0 \%$ & $0 \%$ & $0 \%$ & $0 \%$ & $0 \%$ \\
\hline \multirow{3}{*}{$\begin{array}{l}\text { Somente a } \\
\text { Parede } \\
\text { sofre } \\
\text { alterações }\end{array}$} & 4 & $34,2 \%$ & $54,7 \%$ & $0 \%$ & $0 \%$ & $0 \%$ & $0 \%$ & $0 \%$ \\
\hline & 5 & $34,1 \%$ & $53,6 \%$ & $0 \%$ & $0 \%$ & $0 \%$ & $0 \%$ & $0 \%$ \\
\hline & 6 & $34,2 \%$ & $50,6 \%$ & $0 \%$ & $0 \%$ & $0 \%$ & $0 \%$ & $0 \%$ \\
\hline \multirow{3}{*}{$\begin{array}{l}\text { Somente o } \\
\text { Teto sofre } \\
\text { alterações }\end{array}$} & 7 & $34,1 \%$ & $54,5 \%$ & $0 \%$ & $0 \%$ & $0 \%$ & $0 \%$ & $0 \%$ \\
\hline & 8 & $34,1 \%$ & $53,9 \%$ & $0 \%$ & $0 \%$ & $0 \%$ & $0 \%$ & $0 \%$ \\
\hline & 9 & $34,2 \%$ & $54,9 \%$ & $0 \%$ & $0 \%$ & $0 \%$ & $0 \%$ & $0 \%$ \\
\hline
\end{tabular}

Fonte: as autoras.

A Tabela 12 mostra que para o Céu 12 (claro) há um comportamento similar quando se alteram as superfícies refletoras do teto, parede e piso. Em todos os modelos propostos os percentuais mais elevados encontram-se no ponto $3(1,5 \mathrm{~m}$ da abertura), e reduzem a medida que os pontos se distanciam da abertura. Isto ocorre porque o ponto $1(0,5 \mathrm{~m}$ da abertura) e ponto $2(1,0 \mathrm{~m}$ da abertura) possuem a complementação dos percentuais das UDI no intervalo $500 \leq \mathrm{UDI}<2000 \mathrm{~lx}$.

Tabela 12. Porcentagem diária no intervalo de UDI (Useful Daylight Illuminances) entre 100 lx a 500 lx para o Céu 12 (claro) em função da refletância das superfícies do ambiente interno.

\begin{tabular}{|c|c|c|c|c|c|c|c|c|}
\hline \multicolumn{9}{|c|}{ Percentual de horas diárias no intervalo entre $100 \leq \mathrm{UDI}<500$ lx para o Céu 12 (claro) } \\
\hline \multirow{2}{*}{\multicolumn{2}{|c|}{ Modelos }} & \multicolumn{7}{|c|}{ Pontos de avaliação no ambiente } \\
\hline & & 1 & 2 & 3 & 4 & 5 & 6 & 7 \\
\hline \multirow{3}{*}{$\begin{array}{l}\text { Somente o } \\
\text { Piso sofre } \\
\text { alterações }\end{array}$} & 1 & $14,0 \%$ & $51,7 \%$ & $91,7 \%$ & $37,2 \%$ & $6,2 \%$ & $2,1 \%$ & $0 \%$ \\
\hline & 2 & $15,4 \%$ & $51,5 \%$ & $92,7 \%$ & $36,3 \%$ & $12,3 \%$ & $2,2 \%$ & $2,0 \%$ \\
\hline & 3 & $13,1 \%$ & $45,5 \%$ & $93,6 \%$ & $45,4 \%$ & $12,6 \%$ & $2,0 \%$ & $0 \%$ \\
\hline \multirow{3}{*}{$\begin{array}{l}\text { Somente a } \\
\text { Parede } \\
\text { sofre } \\
\text { alterações }\end{array}$} & 4 & $14,2 \%$ & $55,6 \%$ & $91,8 \%$ & $45,0 \%$ & $11,9 \%$ & $1,8 \%$ & $0 \%$ \\
\hline & 5 & $14,0 \%$ & $51,7 \%$ & $91,7 \%$ & $37,2 \%$ & $6,2 \%$ & $2,1 \%$ & $0 \%$ \\
\hline & 6 & $12,2 \%$ & $54,4 \%$ & $89,2 \%$ & $42,8 \%$ & $11,1 \%$ & $1,5 \%$ & $0 \%$ \\
\hline \multirow{3}{*}{$\begin{array}{l}\text { Somente o } \\
\text { Teto sofre } \\
\text { alterações }\end{array}$} & 7 & $13,9 \%$ & $52,8 \%$ & $90,2 \%$ & $46,4 \%$ & $13,1 \%$ & $0,4 \%$ & $0 \%$ \\
\hline & 8 & $12,2 \%$ & $55,9 \%$ & $91,0 \%$ & $44,8 \%$ & $14,3 \%$ & $1,1 \%$ & $0 \%$ \\
\hline & 9 & $12,0 \%$ & $49,7 \%$ & $92,5 \%$ & $47,0 \%$ & $8,0 \%$ & $0,2 \%$ & $0 \%$ \\
\hline
\end{tabular}

Fonte: as autoras.

Verifica-se na Tabela 13, que para todos os modelos de refletância testados para o Céu 12 (claro), só existem percentuais significativos de horas no intervalo das UDI entre $500 \mathrm{~lx}$ e $2000 \mathrm{~lx}$, até o ponto 2 (1,0m da abertura), mantendo um comportamento similar quando se alteram as superfícies refletoras do teto, parede e piso. Ou seja, a variação das refletâncias internas não ocasiona mudanças significativas neste intervalo de valores das UDI. 
Tabela 13. Porcentagem diária no o intervalo de UDI (Useful Daylight Illuminances) entre 500 lx a 2000 lx para o Céu 12 (claro) em função da refletância das superfícies do ambiente interno.

\begin{tabular}{|c|c|c|c|c|c|c|c|c|}
\hline \multicolumn{9}{|c|}{ 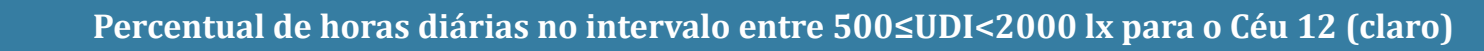 } \\
\hline \multirow{2}{*}{\multicolumn{2}{|c|}{ Modelos }} & \multicolumn{7}{|c|}{ Pontos de avaliação no ambiente } \\
\hline & & 1 & 2 & 3 & 4 & 5 & 6 & 7 \\
\hline \multirow{3}{*}{$\begin{array}{c}\text { Somente o } \\
\text { Piso sofre } \\
\text { alterações }\end{array}$} & 1 & $76,2 \%$ & $48,0 \%$ & $0 \%$ & $0 \%$ & $0 \%$ & $0 \%$ & $0 \%$ \\
\hline & 2 & $74,7 \%$ & $48,1 \%$ & $0 \%$ & $0 \%$ & $0 \%$ & $0 \%$ & $0 \%$ \\
\hline & 3 & $76,5 \%$ & $54,1 \%$ & $0 \%$ & $0 \%$ & $0 \%$ & $0 \%$ & $0 \%$ \\
\hline \multirow{3}{*}{$\begin{array}{l}\text { Somente a } \\
\text { Parede } \\
\text { sofre } \\
\text { alterações }\end{array}$} & 4 & $76,2 \%$ & $44,0 \%$ & $0,1 \%$ & $0 \%$ & $0 \%$ & $0 \%$ & $0 \%$ \\
\hline & 5 & $76,2 \%$ & $48,0 \%$ & $0 \%$ & $0 \%$ & $0 \%$ & $0 \%$ & $0 \%$ \\
\hline & 6 & $78,2 \%$ & $45,3 \%$ & $0 \%$ & $0 \%$ & $0 \%$ & $0 \%$ & $0 \%$ \\
\hline \multirow{3}{*}{$\begin{array}{l}\text { Somente o } \\
\text { Teto sofre } \\
\text { alterações }\end{array}$} & 7 & $76,4 \%$ & $46,9 \%$ & $2,1 \%$ & $0 \%$ & $0 \%$ & $0 \%$ & $0 \%$ \\
\hline & 8 & $78,1 \%$ & $43,8 \%$ & $0,2 \%$ & $0 \%$ & $0 \%$ & $0 \%$ & $0 \%$ \\
\hline & 9 & $78,3 \%$ & $50,0 \%$ & $0 \%$ & $0 \%$ & $0 \%$ & $0 \%$ & $0 \%$ \\
\hline
\end{tabular}

Fonte: as autoras.

\section{Conclusão}

A proposta da pesquisa foi avaliar a interferência que a variação na refletância das superfícies internas de um determinado ambiente, ocasiona na distribuição da iluminação natural do ambiente interno, considerando como fixas a configuração urbana, cujos edifícios vizinhos conformam-se como elementos obstruidores para a entrada da luz natural.

No que se refere às alterações das superfícies refletoras internas, a curva isolux ao longo da profundidade apresenta as maiores alterações nos pontos próximos à abertura, quando são alteradas as características das superfícies refletoras do ambiente.

Observa-se que para o Céu 3 (encoberto), alterar os valores das refletâncias do ambiente pouco contribuiu para variações na média anual de iluminância interna nos pontos da curva isolux.

Para o Céu 7 (parcialmente nublado), alterar os valores das refletâncias do ambiente, parede e piso, contribuiu para a interferência na média anual de iluminância interna nos pontos da curva isolux próximos à abertura, em específico o ponto 2 (1,0 $\mathrm{m}$ da abertura). Para o Céu 12 (claro), alterar os valores das refletâncias do ambiente contribuiu para alteração na média anual de iluminância interna nos pontos da curva isolux próximos à abertura, até o ponto $4(2,0 \mathrm{~m})$. A partir daí as alterações foram insignificantes.

Com relação aos percentuais de horas diárias nos intervalos das UDI entre $500 \mathrm{~lx}$ e $2000 \mathrm{~lx}$ (suficiente) e entre $100 \mathrm{~lx}$ a $500 \mathrm{~lx}$ (suficiente mas com necessidade de complementação com iluminação artificial), a variação da refletância das superfícies internas não ocasionou interferências relevantes para os Céus 3, 7 e 12.

Considerando os resultados obtidos nas simulações, pode-se concluir que a curva isolux ao longo da profundidade apresenta os maiores valores de iluminância nos pontos próximos à abertura. $\mathrm{A}$ variação das superfícies refletoras internas, piso, teto e parede traz pequenas melhorias na iluminação do ambiente interno em pontos próximos da abertura. A medida que os pontos se afastam da abertura não há resultados relevantes de melhoria na distribuição da luz natural no ambiente quando são modificadas as características das superfícies refletoras do ambiente interno. 
Dando prosseguimento ao estudo, prevê-se o seu aprofundamento através da investigação da interferência que o ambiente interno sofre ao serem variadas as superfícies refletoras externas das edificações que compõem o cenário urbano em que está inserido, mantendo fixos os valores das refletâncias internas do ambiente.

\section{Referências}

Araújo, I.A.L., \& Cabús, R.C. (2007). Influência da luz natural refletida pelo entorno na iluminação de edifícios em cânions urbanos no trópico úmido. Anais do Encontro Nacional de Conforto no Ambiente Construído, 9., e do Encontro Latino-Americano de Conforto no Ambiente Construído, 5., Ouro Preto, MG, Brasil.

Cabús, R.C. (2006). TropLux, versão 3: Guia do Usuário. Maceió: Grilu.

Cabús, R.C. (2005). TropLux: um sotaque tropical na simulação da luz natural em edificações. Anais do Encontro Nacional de Conforto no Ambiente Construído, 8., e do Encontro Latino-Americano de Conforto no Ambiente Construído, 4., Maceió, AL, Brasil.

Capeluto, I.G. (2003, May). The influence of the urban environment on the availability of daylighting in office buildings in Israel. Building and Environment, 38(5), 745-752.

Commission Internationale L'aclairage [CIE]. (2003). Spatial distribution of daylight - CIE standard general sky. Viena, Áustria: Publication CIE S 011/E: 2003.

Didoné, E.L. (2009). A influência da luz natural na avaliação da eficiência energética de edifícios contemporâneos de escritórios em Florianópolis/SC. 2009. Dissertação de Mestrado, Universidade Federal de Santa Catarina, Florianópolis, SC, Brasil.

Hopkinson, R.G., Petherbridge, P., \& Longmore, J. (1975). Iluminação natural. Lisboa: Fundação Calouste Gulbenkian.

Iwashita, J. (1999). Estudo da iluminação natural em edifícios de escritórios na cidade de São Paulo Estudo de casos. Anais do Encontro Nacional de Conforto no Ambiente Construido, 5., Fortaleza, CE, Brasil.

Laranja, A.C. Parâmetros urbanos e a disponibilidade de iluminação natural no ambiente interno. (2010). Tese de Doutorado, Universidade Federal do Rio de Janeiro, Rio de Janeiro, RJ, Brasil.

Laranja, A.C., Gazzaneo, L.M.C., \& Cabús, R.C. (2009). Interferências da largura das vias na disponibilidade de iluminação natural do ambiente interno. Labor \& Engenho, 3(1), 55-69. < http://

periodicos.bc.unicamp.br/ojs/index.php/labore/article/view/1851>.

Laranja, A.C., Alvarez, C.E. de, \& Matarangas, K. (2013). Análise da influência da orientação das aberturas na disponibilidade de iluminação natural no ambiente interno na extensão de sua profundidade. Labor \& Engenho, 7(1), 84-98. <http://periodicos.bc.unicamp.br/ojs/index.php/labore/article/view/192>.

Laranja, A.C., Alvarez, C.E. de, \& Campos, N. de P. (2014, jan./mar.). Contribuição dos estudos de Alta Qualidade Ambiental à legislação brasileira. Labor \& Engenho, 8(1), 93-99. <http:// periodicos.bc.unicamp.br/ojs/index.php/labore/article/view/226>.

Laranja, A.C., Alvarez, C.E. de, \& Campos, N. de P. (2014, out./dez.). A influência dos afastamentos laterais das edificações na disponibilidade de iluminação natural no ambiente interno. Labor \& Engenho, 8(4), 88-98. <http://periodicos.bc.unicamp.br/ojs/index.php/labore/article/view/200>.

Lei n.o 4821, de 30 de dezembro de 1998. Código de obras. (1988). Institui o Código de Edificações do Município de Vitória e dá outras providências. Vitória, ES: Diário Oficial (p. 72).

Lei n⿳⺈.6. 6.705, de 2006 Plano Diretor Urbano. (2006). Dispõe sobre o desenvolvimento urbano no Município de Vitória, institui o Plano Diretor Urbano e dá outras providências. Vitória, ES: Diário Oficial (p. 48). 
Nabil, A., \& Mardaljevic, J. (2006). Useful daylight illuminances: A replacement for daylight factors. Energy and Buildings, 38, 905-913. London: Elsevier.

NBR 15215-4: iluminação natural: verificação experimental das condições de iluminação interna de edificações: método de medição. (2005). Rio de Janeiro: Associação Brasileira de Normas Técnicas.

Nikiforiadis, F., \& Pitts, A. (2003). 3D digital geometric reconstruction of the urban environment for daylight simulations studies. Proceedings of the International Building Simulation Conference, 8., Eindhoven, Netherlands.

O'Connor, J., Lee, E., Rubinstein, F., \& Selkowitz, S. (1997). Tips for daylight with windows [LBNL report 39945]. Lawrence Berkeley National Laboratory, USA.

Reinhart, C.F. (2005). A Simulation-Based Review of the Ubiquitous Window-Head-Height to Daylit Zone Depth Rule-of-Thumb. Proceedings of the International Building Simulations Conference, 9. (pp. 1011-1018), Montreal, Canada.

Rocha, A.P. de A. (2012). Caracterização do zoneamento da iluminação natural e sua influência no controle da iluminação artificial. Dissertação de Mestrado, Universidade Federal de Santa Catarina, Florianópolis, SC, Brasil. 